\title{
Interhospital transport of children with bronchiolitis by a statewide emergency transport service
}

Kieren P Fahey, Ben Gelbart, Felix Oberender, Jenny Thompson, Tom Rozen, Christopher James, Catriona McLaren, Jonathan Sniderman and Wonie Uahwatanasakul

Bronchiolitis is a leading cause of emergency admission to paediatric intensive care units (PICUs) both in Australia and New Zealand ${ }^{1-3}$ as well as internationally 4 and is a frequent reason for interhospital transport. ${ }^{5}$ The hallmarks of severity include hypoxia and increased respiratory effort and the mainstay of therapy is respiratory support in the form of oxygen and positive pressure support. In children with severe disease, admission to intensive care is required for non-invasive and invasive respiratory support. In Victoria, Australia, paediatric intensive care is centralised and, therefore, children with severe bronchiolitis in hospitals without paediatric intensive care services frequently require emergency transport.

In the past decade, there has been a decline in the proportion of children with bronchiolitis receiving mechanical ventilation. ${ }^{2}$ The high flow nasal cannula (HFNC) has become the predominant respiratory support mode, reducing the proportion of inpatients who require escalation of care.1,6 The key benefits of HFNC is the relative ease of administration, safety, tolerability and the ability to administer it outside of intensive care. However, its use has seen an increased rate of PICU admissions despite no definite change in the absolute number of children who require invasive mechanical ventilation. ${ }^{7}$

Recognition of the deteriorating patient in hospital is a key health care priority. ${ }^{8}$ Children with severe bronchiolitis in ward settings commonly have physiological criteria triggering escalation of care and, therefore, in hospitals without a PICU, interhospital transport is often required. In October 2014, the Victorian Children's Tool for Observation and Response (VICTOR) charts were introduced as a standardised, statewide intervention to help clinicians recognise clinical deterioration and trigger escalation of care. ${ }^{9}$ The impact on children with bronchiolitis and subsequent need for interhospital transport and intensive care support is not known.

We hypothesised that the introduction of HFNC and increased recognition of children with bronchiolitis identified for escalation of care by ViCTOR charts would increase interhospital transport of such children. Accordingly, we performed a retrospective study of emergency transport

\begin{abstract}
Objective: To investigate the rate of interhospital emergency transport for bronchiolitis and intensive care admission following the introduction of high flow nasal cannula and standardised paediatric observation and response charts.

Design: Retrospective cohort study.

Setting: A statewide paediatric intensive care transport service and its two referral paediatric intensive care units (PICUs) in Victoria, Australia.
\end{abstract}

Participants: Children less than 2 years old emergently transported with bronchiolitis during two time periods: 2008-2012 and 2015-2019.

Main outcome measures: Incidence rates of bronchiolitis transport episodes, PICU admissions and respiratory support.

Results: 802 children with bronchiolitis were transported during the study period, 233 in the first period (20082012) and 569 in the second period (2015-2019). The rate of interhospital transport for bronchiolitis increased from 32.9 to 71.8 per 100000 children aged $0-2$ years. The population-adjusted rate of PICU admission increased from 16.2 to 36.6 per 100000 children aged $0-2$ years. Metropolitan hospitals were the predominant referral source and this increased from $60.1 \%$ of transports to $78.6 \%(P<0.001)$. In children admitted to a PICU, the administration of high flow nasal cannula during transport increased significantly from $1.7 \%$ to $75.9 \%(P<0.001)$ and a concomitant reduction in continuous positive airway pressure and mechanical ventilation occurred (40-12.4\% and $27-6.9 \%$ respectively; $P<0.001)$. The proportion of mechanical ventilation as well as PICU and hospital length of stay decreased over time.

Conclusions: The population-adjusted rate of interhospital transport and admission to the PICU for bronchiolitis increased over time. This occurred despite a lower rate of non-invasive and invasive mechanical ventilation during transport and in the PICU.

Crit Care Resusc 2021; 23 (3): 292-99 


\section{ORIGINAL ARTICLE}

episodes by a statewide paediatric emergency transport service over two time periods to investigate whether resource utilisation and intensive care outcome had changed. These time periods were chosen to distinguish a before and after VICTOR chart implementation and the emergence of HFNC therapy.

\section{Methods}

A retrospective cohort study was performed in children with bronchiolitis requiring interhospital transport in Victoria, between 1 January 2008 to 31 December 2012 and 1 January 2015 to 31 December 2019. The primary objective of the study was to compare the rate of interhospital transport between the two time periods. The secondary objectives were to compare the respiratory support characteristics, intensive care support and outcomes in patients who received such support. A 2-year intervening period was excluded to account for the staggered introduction of HFNC and allow for the commencement of ViCTOR charts in Victoria. Approval to conduct the study was provided by the Human Research and Ethics Committee of the Royal Children's Hospital and Monash Children's Hospital (HREC No. QA/65001/RCHM-2020).

In Victoria, paediatric emergency transport is performed by the Paediatric Infant Perinatal Emergency Retrieval (PIPER) service. It is a central referral service based at the Royal Children's Hospital, Melbourne, operating with a control paediatric intensivist and a specialist transport team consisting of a senior paediatric intensive care trainee and a paediatric intensive care nurse. Decisions regarding retrieval, therapy and disposition are the responsibility of the intensivist. In 2015, HFNC was introduced by PIPER. Children weighing less than $5 \mathrm{~kg}$ are transported by the transported by neonatal PIPER. Paediatric intensive care services in Victoria are provided by two tertiary PICUs: the Royal Children's Hospital Melbourne and Monash Children's Hospital.

The number of transport episodes and patient demographic and clinical data were obtained from the PIPER database. These data include transport respiratory support mode, referring hospital location and retrieval duration.

Intensive care unit data were extracted from the respective PICU databases. In addition to age and sex and Paediatric Index of Mortality (PIM) 2 and 3, viral aetiology and additional diagnoses, including congenital heart disease, chronic lung disease, neurological disease, genetic disorders as well as prematurity (defined as $<37$ weeks' gestation at birth), were recorded. Clinical data included the maximum level of respiratory support, hospital and PICU length of stay, and hours of respiratory support.

\section{Inclusion and exclusion criteria}

All transport episodes were included if the diagnosis assigned by the paediatric transport service was bronchiolitis in children less than 2 years old. We excluded children transported by neonatal PIPER or intensive care admissions to mixed adult/paediatric centres. For children admitted to the PICU, only direct admissions following transport were included. Admissions to the PICU via a ward setting were considered a potential confounder, as time from transport to PICU admission may have varied.

\section{Outcomes and definitions}

The primary outcome was the rate of emergency transport of children with bronchiolitis as a proportion of total emergency transports and population adjusted (per 100000 children aged 0-2 years). Secondary outcomes included emergency transport respiratory support modes, rate of PICU admission (as a proportion of all transports and as a proportion of all PICU admissions). For patients admitted to a PICU, respiratory support modes and outcomes such as duration of respiratory support, PICU and hospital length of stay, and mortality were compared.

\section{Statistical analysis}

Categorical data were described using frequencies and proportions and continuous data using median and interquartile range (IQR). Normality of datasets was determined through the Shapiro-Wilk test and visualised with histograms. Wilcoxon rank sum tests were used to compare groups for non-parametric data. The $\chi^{2}$ test, Fischer exact test (as appropriate) and incidence rate ratio (IRR) with $95 \%$ confidence interval $(\mathrm{Cl})$ were used to compare proportions and population incidence. $P$ values of less than 0.05 were considered significant. Referring hospital locations were categorised as either metropolitan or regional based on the Victoria State Government's definition. ${ }^{10}$ The population-adjusted rate was based on Australian Bureau of Statistics data. ${ }^{11}$ Rates are reported per 100000 Victoria Children aged $0-2$ years. A post hoc analysis was performed excluding the year 2015 due to the very high number of transports in that year. Analyses were performed using Stata version 16.1 (StataCorp, Texas, USA).

\section{Results}

There were 802 paediatric emergency transports for bronchiolitis out of 5056 total transports (15.9\%) during the study period. Bronchiolitis transports increased from $233 / 1972$ transports $(11.8 \%)$ to $569 / 3084$ transports $(18.5 \%)$ in the second period $(P<0.001)$. Seventy-three per cent were from metropolitan hospitals. 


\section{ORIGINAL ARTICLE}

\section{Rate of emergency transport}

The rates of emergency transport (including populationadjusted) for the entire cohort and by period are shown in Table 1. The population-adjusted rate of emergency bronchiolitis transports more than doubled between periods, from 32.9 to 71.8 per 100000 children aged $0-2$ years (IRR, 2.2; 95\% Cl, 1.9-2.6; $P<0.001$ ), as did subsequent transport to a PICU, which increased from 16.2 to 36.6 per 100000 children (IRR, 2.3; $95 \% \mathrm{Cl}, 1.8-2.8 ; P<0.001$ ). The rate of emergency transport per 100000 children per year by referral location is shown in Figure 1. Emergency transport from metropolitan hospitals increased in the second period (140[60.1\%] v $447[78.6 \%] ; P<0.001)$ (Table 1).

\section{Sensitivity analysis}

In a post hoc analysis excluding the year 2015, the rate of emergency transport, nonetheless, remained significantly higher in the second period (Online Appendix, supplementary table 1).

\section{Demographic characteristics of admissions to the PICU following emergency transport}

Direct admission to a PICU occurred in 405 out of 802 bronchiolitis transports $(50.5 \%)$ during the study period.
Demographic and clinical characteristics of admissions to the PICU are shown in Table 2. The median age at admission was slightly higher in the second period (226 days [IQR, 114-313.9] $\vee 256$ days [IQR, 146-352]; $P=0.024)$. There was an increase in proportion of admissions to PICU from a metropolitan hospital in the second period (67/115 [58.3\%] v 212/290 [73.1\%]; $P=0.004)$.

\section{Respiratory support during emergency transport}

Respiratory support characteristics for all bronchiolitis transports are shown in the Online Appendix, supplementary table 2. In the second period, the use of HFNC increased $(5 / 233[2.1 \%] \vee 443 / 569$ [77.9\%]; $P<0.001)$ and the rate (including population-adjusted) of mechanical ventilation for all transported children decreased (36 [15.5\%] v 24 [4.2\%]; $P<0.001 ; \mathrm{IRR}, 0.6$ [95\% Cl, 0.3-1.0]; $P=0.049$ ) (Online Appendix, supplementary table 2 ). In children admitted to a PICU, the rate (including population-adjusted) of HFNC increased (2/115 [1.7\%] V 220/290 [75.9\%]; $P<0.001)$, whereas mechanical ventilation decreased (31 [27.0\%] v 20 [6.9\%]; $P<0.001 ;$ IRR, $0.6[95 \% \mathrm{Cl}, 0.3-1.0] ; P=0.054)$ during transport (Table 3 ). Nine mechanically ventilated children with a transport diagnosis of bronchiolitis were subsequently admitted to a neonatal unit or admitted to a PICU with a revised admission diagnosis. Transport

Table 1. Rates of emergency transport for bronchiolitis over the study period

\begin{tabular}{|c|c|c|c|c|c|}
\hline Variable & Entire cohort & 2008-2012 & 2015-2019 & $\begin{array}{l}\text { Incidence rate } \\
\text { ratio }(95 \% \mathrm{Cl})\end{array}$ & $P$ \\
\hline Total Victorian population aged $0-2$ years & 1499767 & 707748 & 792019 & & \\
\hline Total emergency transports & 5056 & 1972 & 3084 & & \\
\hline $\begin{array}{l}\text { Emergency transports per } 100000 \text { population } \\
\text { aged } 0-2 \text { years }\end{array}$ & 337.1 & 278.6 & 389.4 & $1.4(1.3-1.5)$ & $<0.001$ \\
\hline Bronchiolitis emergency transports & 802 & 233 & 569 & & \\
\hline Proportion of all emergency transport, \% & $15.9 \%$ & $11.8 \%$ & $18.5 \%$ & & $<0.001$ \\
\hline Per 100000 population aged $0-2$ years & 53.5 & 32.9 & 71.8 & $2.2(1.9-2.6)$ & $<0.001$ \\
\hline $\begin{array}{l}\text { Admissions to PICU following emergency } \\
\text { transport for bronchiolitis }\end{array}$ & 405 & 115 & 290 & & \\
\hline $\begin{array}{l}\text { Proportion of bronchiolitis emergency } \\
\text { transports, \% }\end{array}$ & $50.5 \%$ & $49.4 \%$ & $51 \%$ & & 0.681 \\
\hline Proportion of all admissions to a PICU, \% & $2 \%$ & $1.4 \%$ & $2.5 \%$ & & $<0.001$ \\
\hline Per 100000 population aged $0-2$ years & 27 & 16.2 & 36.6 & $2.3(1.8-2.8)$ & $<0.001$ \\
\hline \multicolumn{6}{|l|}{$\begin{array}{l}\text { Referring hospital location for all bronchiolitis } \\
\text { transports }\end{array}$} \\
\hline Metropolitan hospital & $587(73.2 \%)$ & $140(60.1 \%)$ & $447(78.6 \%)$ & & $<0.001$ \\
\hline Regional hospital & $215(26.8 \%)$ & $93(39.9 \%)$ & $122(21.4 \%)$ & & \\
\hline
\end{tabular}

PICU = paediatric intensive care unit. 


\section{ORIGINAL ARTICLE}

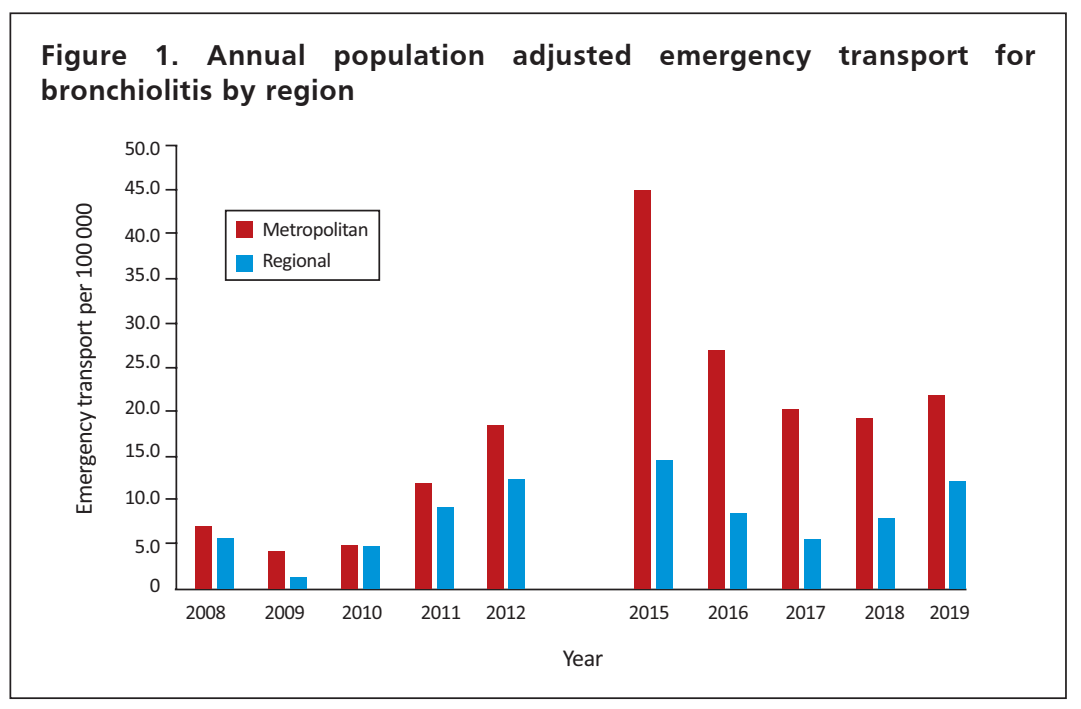

increased in the second period.

Our findings show that more children are being transported for bronchiolitis, yet their requirement for intensive care support has decreased. This may be placing considerable burden on children, their families and health care resources, and because outcomes such as the need for invasive and non-invasive ventilation have improved over time, a number of such transports may potentially be avoidable.

Several factors may explain our findings. The introduction of statewide observation and response charts in Victoria in 2014 is a crucial intervention to standardise and optimise the recognition of clinical deterioration and critical illness in hospitalised children. It is possible

respiratory support modes per year are shown in the Online Appendix, supplementary figure.

\section{Respiratory support during intensive care admission and outcomes}

Mechanical ventilation was reduced in the second period, both as a proportion (77/115 [67\%] v 56/290 [19.3\%]; $P<0.001)$ and population-adjusted, from 10.9 to 7.1 per 100000 population (IRR, $0.6[95 \% \mathrm{Cl}, 0.5-0.9] ; P=0.014)$ (Table 4). Non-invasive ventilation (including continuous positive airway pressure and HFNC) increased threefold in the second period (22/115 [19.1\%] v 220/290 [75.9\%]; $P<0.001)$. Respiratory support hours, PICU and hospital length of stay were also reduced. There were no deaths over the study period.

\section{Discussion}

\section{Key findings}

In the second period, emergency transport for bronchiolitis as a proportion of all transports increased by over $50 \%$ and more than doubled per 100000 Victorian children. However, during transport, the number and proportion requiring invasive ventilation decreased while HFNC use substantially increased. The proportion of transport episodes subsequently admitted to a PICU did not change between periods, but the population-adjusted rate more than doubled. The proportion of all children receiving mechanical ventilation during transport decreased, as did the population-adjusted rate. So too did the proportion of children who received mechanical ventilation in the PICU. Respiratory support hours and PICU and hospital length of stay all decreased in the second period. Metropolitan hospitals were the most common referral source and this that statewide observation and response charts identified abnormal physiological signs more frequently in settings where intensive care resources were unavailable therefore triggering interhospital transport. The lack of paediatric high dependency or intensive care services at referring hospitals may be a reason for increased interhospital transport. Our importance. Lower illness severity in the second period could also explain the differences. We cannot compare severity of illness between periods. Importantly, there was no clinically significant difference in age nor difference in presence of additional diagnoses. There was a lower proportion of chronic lung disease in the second period despite a stable incidence of bronchopulmonary dysplasia in preterm neonates in Australia and New Zealand over the past decade (to $9.8 \%$ in 2019). ${ }^{12}$ Although the PIM2/ PIM3 score was higher in the first period, it is not a suitable marker of bronchiolitis severity at presentation.

The emergence of HFNC for children with bronchiolitis is another possible explanation for increased interhospital transports. HFNC is a safe, well tolerated ${ }^{13}$ and less invasive mode of respiratory support. It provides oxygenation and some positive pressure support and can be administered in a ward setting. ${ }^{14,15}$ For children in intensive care with bronchiolitis, it may reduce the subsequent need for mechanical ventilation. ${ }^{16}$ Previous studies of the effect reduction in the proportion (but not absolute numbers) of children requiring mechanical ventilation. ${ }^{17}$ Our study shows a reduction in the number, proportion and population-adjusted rate of mechanical ventilation in the second period, but we cannot make any causal inferences about either of these interventions.

Paediatric early warning tools have enabled the early recognition and subsequent intervention for children who data cannot confirm these factors nor indicate their relative of HFNC during paediatric emergency transport show a 


\begin{tabular}{|c|c|c|c|c|}
\hline Variable & All cohort & 2008-2012 & 2015-2019 & $P$ \\
\hline Total episodes & 405 & 115 & 290 & \\
\hline Age (days), median (IQR) & $248(131.4-339.5)$ & $226(114-313.9)$ & $256(146-352)$ & 0.024 \\
\hline Neonates, 0-28 days & 0 & 0 & 0 & \\
\hline 29-90 days & 53 & 20 & 33 & \\
\hline 91 days to 1 year & 278 & 81 & 197 & \\
\hline $1-2$ years & 74 & 14 & 60 & \\
\hline $\mathrm{PIM}^{*}$ probability of death (\%), median (IQR) & $0.16 \%(0.14-0.38 \%)$ & $0.42 \%(0.20-0.63 \%)$ & $\begin{array}{c}0.15 \%(0.14- \\
0.23 \%)\end{array}$ & $<0.001$ \\
\hline Sex, male & $237(58.5 \%)$ & $70(60.9 \%)$ & $167(57.6 \%)$ & 0.543 \\
\hline \multicolumn{5}{|l|}{ Additional diagnosis } \\
\hline Any additional diagnosis & $138(34.1 \%)$ & $44(38.3 \%)$ & $94(32.4 \%)$ & 0.259 \\
\hline Congenital heart disease & $56(13.8 \%)$ & $18(15.7 \%)$ & $38(13.1 \%)$ & 0.494 \\
\hline Chronic lung disease & $39(9.6 \%)$ & $19(16.5 \%)$ & $20(6.9 \%)$ & 0.003 \\
\hline Neurological disease & $10(2.5 \%)$ & $6(5.2 \%)$ & $4(1.4 \%)$ & 0.035 \\
\hline Prematurity (< 37 weeks) & $97(27.4 \%)$ & $29(31.2 \%)^{\dagger}$ & $68(26.1 \%)^{\ddagger}$ & 0.344 \\
\hline Genetic disorders & $18(4.4 \%)$ & $5(4.4 \%)$ & $13(4.5 \%)$ & 0.965 \\
\hline \multicolumn{5}{|l|}{ Viral aetiology } \\
\hline All viruses & 284 & $61(53.0 \%)$ & $223(76.9 \%)$ & $<0.001$ \\
\hline RSV & $164(40.5 \%)$ & $42(36.5 \%)$ & $122(42.1 \%)$ & \\
\hline hMPV & $23(5.7 \%)$ & $3(2.6 \%)$ & $20(6.9 \%)$ & \\
\hline Influenza & $7(1.7 \%)$ & $1(0.9 \%)$ & $6(2.1 \%)$ & \\
\hline Parainfluenza & $14(3.5 \%)$ & $1(0.9 \%)$ & $13(4.5 \%)$ & \\
\hline Adenovirus & $13(3.2 \%)$ & $1(0.9 \%)$ & $12(4.1 \%)$ & \\
\hline Picornavirus (including rhinovirus) & $63(15.6 \%)$ & $13(11.3 \%)$ & $50(17.2 \%)$ & \\
\hline \multicolumn{5}{|l|}{ Referring hospital region } \\
\hline Metropolitan hospital & 279 (68.9\%) & $67(58.3 \%)$ & $212(73.1 \%)$ & 0.004 \\
\hline Regional hospital & $126(31.1 \%)$ & $48(41.7 \%)$ & $78(26.9 \%)$ & \\
\hline
\end{tabular}

hMPV = human metapneumovirus; IQR = interquartile range; PICU = paediatric intensive care unit; PIM = Paediatric Index of Mortality; RSV = respiratory syncytial virus. * PIM3 after 2013, PIM2 before 2013. † From 93 known values. ₹ From 261 known values.

deteriorate in hospital, $18-20$ but bronchiolitis-specific tools do not exist. The sensitivity of such tools can vary according to underlying conditions. ${ }^{21}$ No such modifications have been described in hospitalised children with bronchiolitis. Vital sign thresholds are commonly used to grade severity in bronchiolitis; however, standard vital sign thresholds may indicate the need for escalation of care but not the need for mechanical ventilation.

A combination of these factors likely contributed to our findings. As HFNC emerged, development of hospital processes to accommodate such therapy outside of a PICU may have lagged. Our data indicate that an initial spike in transports in 2015 subsequently reduced and stabilised in subsequent years. Sensitivity analysis was consistent with the primary analysis.

\section{Implications of study findings}

The increased rate of transport of children with bronchiolitis and the reduced requirement for mechanical ventilation imply that better systems could facilitate the care of such children in hospitals without paediatric intensive care services. Improved methods for identifying children at risk of requiring higher levels of respiratory support are needed. The challenge in the management of bronchiolitis in centres 


\begin{tabular}{|c|c|c|c|c|}
\hline Total respiratory therapies during transport & 115 & 290 & & \\
\hline No oxygen & $2(1.7 \%)$ & $8(2.76 \%)$ & & 0.731 \\
\hline Low flow oxygen & $34(29.6 \%)$ & $6(2.1 \%)$ & & $<0.001$ \\
\hline Per 100000 population & 4.8 & 0.8 & $0.2(0.1-0.4)$ & $<0.001$ \\
\hline HFNC & $2(1.7 \%)$ & $220(75.9 \%)$ & & $<0.001$ \\
\hline Per 100000 population & 0.3 & 27.8 & $98.3(26.9-816.7)$ & $<0.001$ \\
\hline Per 100000 population & 4.4 & 2.5 & $0.6(0.3-1.0)$ & 0.054 \\
\hline
\end{tabular}

\begin{tabular}{|c|c|c|c|c|}
\hline Variable & 2008-2012 & 2015-2019 & $\begin{array}{l}\text { Incidence rate ratio } \\
(95 \% \mathrm{Cl})\end{array}$ & $P$ \\
\hline Admissions to PICU & 115 & 290 & & \\
\hline \multicolumn{5}{|l|}{ Respiratory therapies in PICU } \\
\hline Low flow oxygen & $16(13.9 \%)$ & $14(4.8 \%)$ & & 0.002 \\
\hline - Per 100000 population & 2.3 & 1.8 & $0.78(0.4-1.7)$ & 0.507 \\
\hline HFNC/CPAP & $22(19.1 \%)$ & $220(75.9 \%)$ & & $<0.001$ \\
\hline - Per 100000 population & 3.1 & 27.8 & $8.9(5.8-14.6)$ & $<0.001$ \\
\hline Mechanical ventilation & $77(67.0 \%)$ & $56(19.3 \%)$ & & $<0.001$ \\
\hline - Per 100000 population & 10.9 & 7.1 & $0.6(0.5-0.9)$ & 0.014 \\
\hline \multicolumn{5}{|l|}{$\begin{array}{l}\text { Duration of respiratory support and PICU and } \\
\text { hospital LOS (h), median (IQR) }\end{array}$} \\
\hline Respiratory support (all) & $43(15.6-71.5)$ & $22.5(13.6-40.8)$ & & $<0.001$ \\
\hline Mechanical ventilation & $54.3(25.7-92)$ & $41.9(23.1-82.8)$ & & 0.312 \\
\hline PICU LOS (all) & $56.8(32.1-106.2)$ & $41.4(24.7-64.0)$ & & $<0.001$ \\
\hline PICU LOS (mechanical ventilation) & $89.3(52-117.6)$ & $79.3(59.2-138.8)$ & & 0.886 \\
\hline Hospital LOS (all) & $116.7(73.5-179.1)$ & $71.7(47.3-111.6)$ & & $<0.001$ \\
\hline Hospital LOS (mechanical ventilation) & $154(97.5-219.6)$ & $107(74.4-176.5)$ & & 0.042 \\
\hline
\end{tabular}

without PICUs is establishing systems to enable ward-based management of HFNC and optimally recognising those children who require higher levels of respiratory support. This would improve the impact on children, their families and health care resources.

\section{Strengths and limitations}

The strengths of this study include the use of statewide data from a centralised transport service and from two state referral paediatric intensive care services. The study period spans more than a decade and allows for the effect 


\section{ORIGINAL ARTICLE}

of introducing track-and-trigger charts and HFNC to be examined. However, some limitations should be noted. Very young infants were excluded as we did not include data from the neonatal transport service, aiming instead to investigate the specific effects on paediatric transport and paediatric intensive care services. The retrospective nature of our study prevents making causal associations and warrants careful consideration of confounding factors. We do not present population incidence of bronchiolitis nor hospitalisation rates for bronchiolitis which may influence our results, but we include population-adjusted rates providing a suitable denominator. We could not analyse illness severity at presentation. These data are not reported and, in general, such scores are variable in outcome prediction. ${ }^{22}$ We therefore could not analyse the results adjusting for illness severity but do present median age and PIM2/PIM3 score in both periods. We did not have access to the total number of bronchiolitis hospitalisations for the whole period and also did not include children admitted to the PICU with an intervening period on the ward. Changes in health services over time, for example, may have influenced the referral rate and are not accounted for. Development of high dependency units and intensive care services for children in regional areas and mixed adult/paediatric centres took place during the study periods, potentially affecting the results. However, any such impact would likely be a reduction in the number of transports to intensive care. Two randomised controlled studies for bronchiolitis were conducted in Victoria during the second period: the PARIS (ACTRN12613000388718) and the DAB studies (ACTRN12613000316707). ${ }^{6}$ Neither study administered interventions during transport and neither were conducted at referring hospitals. Therefore, these studies would not have affected transport but they may have had an impact on intensive care resource utilisation. We were unable to differentiate continuous positive airway pressure and HFNC from some PICU respiratory support data as both are categorised as non-invasive ventilation. However, the rate of mechanical ventilation was able to be differentiated. We were unable to determine the criteria for referral, transport or PICU admission from our data. We did not account for children admitted to a paediatric ward after transport who were subsequently admitted to a PICU.

\section{Conclusions}

The population-adjusted rate of emergency transport of children with bronchiolitis has increased over time. The population-adjusted rate of PICU admission increased despite a decrease in the need for invasive respiratory support. HFNC use during transport substantially increased. The rate of interhospital transport from metropolitan hospitals increased over time.
Acknowledgements: We thank Sarah Wolfe, Carmel Delzoppo and Megan Harvey for their assistance with data collection.

\section{Competing interests}

No relevant disclosures.

\section{Author details}

Kieren P Fahey 1,2

Ben Gelbart ${ }^{1,3,4}$

Felix Oberender ${ }^{5,6}$

Jenny Thompson ${ }^{1}$

Tom Rozen ${ }^{1,2,4}$

Christopher James ${ }^{1,2,4}$

Catriona McLaren ${ }^{5}$

Jonathan Sniderman ${ }^{7}$

Wonie Uahwatanasakul ${ }^{2}$

1 Paediatric Intensive Care Unit, Royal Children's Hospital Melbourne, Melbourne, VIC, Australia.

2 Department of Paediatrics, University of Melbourne, Melbourne, VIC, Australia.

3 Department of Paediatrics, Department of Critical Care, University of Melbourne, Melbourne, VIC, Australia.

4 Murdoch Children's Research Institute, Melbourne, VIC, Australia.

5 Paediatric Intensive Care Unit, Monash Children's Hospital, Melbourne, VIC, Australia.

6 Department of Paediatrics, School of Clinical Sciences, Monash University, Melbourne, VIC, Australia.

7 Department of Critical Care, Paediatrics, Schulich School of Medicine and Dentistry, London, On, Canada.

Correspondence: Ben.gelbart@rch.org.au

doi: https://doi.org/10.51893/2021.3.0A6

\section{References}

1 Schlapbach LJ, Straney L, Gelbart B, et al. Burden of disease and change in practice in critically ill infants with bronchiolitis. Eur Respir J 2017; 49:1601648.

2 Ganu SS, Gautam A, Wilkins B, Egan J. Increase in use of non-invasive ventilation for infants with severe bronchiolitis is associated with decline in intubation rates over a decade. Intensive Care Med 2012; 38: 1177-83.

3 Ghazaly M, Nadel S. Characteristics of children admitted to intensive care with acute bronchiolitis. Eur J Pediatr 2018; 177: 913-20.

4 Fujiogi M, Goto T, Yasunaga $H$, et al. Trends in bronchiolitis hospitalizations in the United States: 2000-2016. Pediatrics 2019; 144: e20192614.

5 Ramnarayan P, Dimitriades K, Freeburn L, et al. Interhospital transport of critically ill children to PICUs in the United 


\section{ORIGINAL ARTICLE}

Kingdom and Republic of Ireland: analysis of an international dataset. Pediatr Crit Care Med 2018; 19: e300-11.

6 Franklin D, Babl FE, Schlapbach LJ, et al. A randomized trial of high-flow oxygen therapy in infants with bronchiolitis. N Engl J Med 2018; 378: 1121-31.

7 Pham H, Thompson J, Wurzel D, et al. Ten years of severe respiratory syncytial virus infections in a tertiary paediatric intensive care unit. J Paediatr Child Health 2020; 56: 61-7.

8 Australian Commission on Safety and Quality in Health Care. National consensus statement: essential elements for recognising and responding to clinical deterioration; 2 nd ed. Sydney: ACSQHC, 2017. https://www.safetyandquality.gov. au/sites/default/files/migrated/National-Consensus-Statementclinical-deterioration_2017.pdf (viewed July 2021).

9 ViCTOR. Victorian Children's Tool for Observation and Response (ViCTOR). https://www.victor.org.au (viewed Feb 2020).

10 Regional Development Victoria. Victoria's regions. https:// www.rdv.vic.gov.au/victorias-regions\#region-listing (viewed June 2020).

11 Australian Bureau of Statistics. Australian demographic statistics, Sept 2019. https://www.abs.gov.au/ausstats/abs@. nsf/mf/3101.0 (viewed Mar 2020).

12 Chow SSW, Creighton P, Chambers GM, Lui, K. Report of the Australian and New Zealand Neonatal Network 2019. Sydney: ANZNN, 2021. https://anznn.net/Portals/O/AnnualReports/ Report\%20of\%20the\%20Australian\%20and\%20New\%20 Zealand\%20Neonatal\%20Network\%202019.pdf (viewed July 2021).

13 Anil S, Rohit V, Gupta N, et al. Comparison of high-flow nasal cannula and noninvasive positive pressure ventilation in children with acute bronchiolitis. J Pediatr Crit Care 2020; 7: 168-73.

14 Kallappa C, Hufton M, Millen G, et al. Use of high flow nasal cannula oxygen (HFNCO) in infants with bronchiolitis on a paediatric ward: a 3-year experience. Arch Dis Child 2014; 99: 790-1.

15 Kalburgi S, Halley T. High-flow nasal cannula use outside of the ICU setting. Pediatrics 2020; 146: 1-7.

16 Schibler A, Pham TM, Dunster KR, et al. Reduced intubation rates for infants after introduction of high-flow nasal prong oxygen delivery. Intensive Care Med 2011; 37: 847-52.

17 Schlapbach LJ, Schaefer J, Brady AM, et al. High-flow nasal cannula (HFNC) support in interhospital transport of critically ill children. Intensive Care Med 2014; 40: 592-9.

18 Brady PW, Muething S, Kotagal U, et al. Improving situation awareness to reduce unrecognized clinical deterioration and serious safety events. Pediatrics 2013; 131: e298-308.

19 Tibballs J, Kinney S. Reduction of hospital mortality and of preventable cardiac arrest and death on introduction of a pediatric medical emergency team. Pediatr Crit Care Med 2009; 10: 306-12.

20 Bonafide $C P$, Localio $A R$, Roberts $K E$, et al. Impact of rapid response system implementation on critical deterioration events in children. JAMA Pediatr 2014; 168: 25-33.

21 Dean NP, Fenix JB, Spaeder M, et al. Evaluation of a pediatric early warning score across different subspecialty patients. Pediatr Crit Care Med 2017; 18: 655-60.

22 Rodriguez-Martinez CE, Sossa-Briceño MP, Nino G. Systematic review of instruments aimed at evaluating the severity of bronchiolitis. Paediatr Respir Rev 2018; 25: 43-57. 